\title{
A non-linear control method for active magnetic bearings with bounded input and output
}

\author{
Danh Huy Nguyen, Tung Lam Nguyen, Duc Chinh Hoang \\ School of Electrical Engineering, Hanoi University of Science and Technology, Vietnam
}

\begin{abstract}
Article Info
Article history:

Received Apr 24, 2020

Revised Jun 10, 2020

Accepted Jul 28, 2020

\section{Keywords:}

Active magnetic bearing Backstepping control Barrier Lyapunov function Input saturation

ABSTRACT

Magnetic bearing is well-known for its advantage of reducing friction in rotary machines and is placing conventional bearings where high-speed operations and cleanliness are essential. It can be shown that the AM is a nonlinear system due to the relation between the magnetic force and current/rotor displacement. In this paper, a mathematical model of a 4-DOF AMB supported by four dual electric magnets is presented. The control objective is placed in a view of control input saturation and output limitation that are meaningful aspect in practical applications. Backstepping algorithm based control strategy is then adopted in order to achieve the high dynamic performance of the bearing. The control is designed in such a way that it takes input and output constraints into account by flexibly using hyperbolic tangent and barrier Lyapunov functions. Informative simulation studies are carried out to understand the operations of the machine and evaluate the controller quality.
\end{abstract}

This is an open access article under the CC BY-SA license.

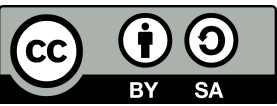

\section{Corresponding Author:}

Tung Lam Nguyen,

School of Electrical Engineering,

Hanoi University of Science and Technology, Vietnam.

Email: lam.nguyentung@hust.edu.vn

\section{INTRODUCTION}

$\mathrm{AMB}$ is one of the magnetic suspension methods which enables rotor shaft in rotary machines to be lifted off without mechanical support by actively controlling the electromagnet [1]. Absence of mechanical contact results in friction reduction. Thus, speed or acceleration of supported components can be increased significantly when comparing with conventional bearing in use. Authors in [2] has listed many advantages of AMB such as the absence of lubrication and contaminating wear, high speed rotation, low bearing losses, etc. These benefits allow AMB to be integrated into electrical motor which can rotate upto $200000 \mathrm{rpm}$ [3] and be applicable as ultra-high speed spindle in machine tools [4, 5]. AMB can also be adopted in vacuum and cleanroom systems [6] or equipment with harsh working condition like turbo machinery [7, 8]. Design and different structures of magnetic bearings have been presented in $[2,9]$. It can be seen that the system is highly non-linear, and can become very complex. Thus the challenge lies in developing control scheme of bearing so that it assures high performance features, especially nanometer accuracy [10] since the gap between rotor shaft and bearings can be extremely tiny. Poor design of controller may result in rotor unbalances and internal damping which in turns create vibration in machines, crack in motor shaft and failure at the end [11, 12]. The classical PID algorithm have been widely used to control AMB system due to its simplicity, adaptability and maturity $[13,14]$. As AMB is a non-linear system, other methods like feedback linearisation or sliding mode control can also be applied $[10,15]$. With an effort to eliminate uncertainties in plant modelling, Bonffito et al propose an offset free control for AMB based on classical model predictive control [16]. 
It is evident that in previously mentioned research, hard limitation on control input and output is omitted [17-20]. Control saturation might result in control degradation and violation of output constraint leads to system mechanical failure. In this paper, we have adopted backstepping control algorithm to regulate and stabilize the operation of AMB. The backstepping method há been employed in robotics [21], process control [22], space applications [23, 24], and in AMB systems [25]. It is proven to be suitable with strict-feedback system and to have the flexibility of removing instability while avoiding cancellation of potentially useful nonlinearities [21]. The contribution of the paper can be named is the consideration of bounded system input and output in control design. This paper is organized as the following. The mathematical model of AMB is first developed in Section 2. Controller design process is presented in Section 3. In Section 4. simulation results are provided together with the discussions. Finally, Section 5. concludes this paper.

\section{MATHEMATICAL MODEL OF A DUEL COILS MAGNETIC ACTUATOR}

It is fundamental that the magnetic force is proportional to the current square. Thus, regulating the current can result in the force change. It is assumed that the rotor shaft has already been elevated along $\mathrm{z}$ axis in vertical direction by another system. The system includes 2 pairs of the same electromagnets along $x$ and $y$ axes in horizontal directions, ones of each pair are placed in the opposite position as illustrated in Figure 1.

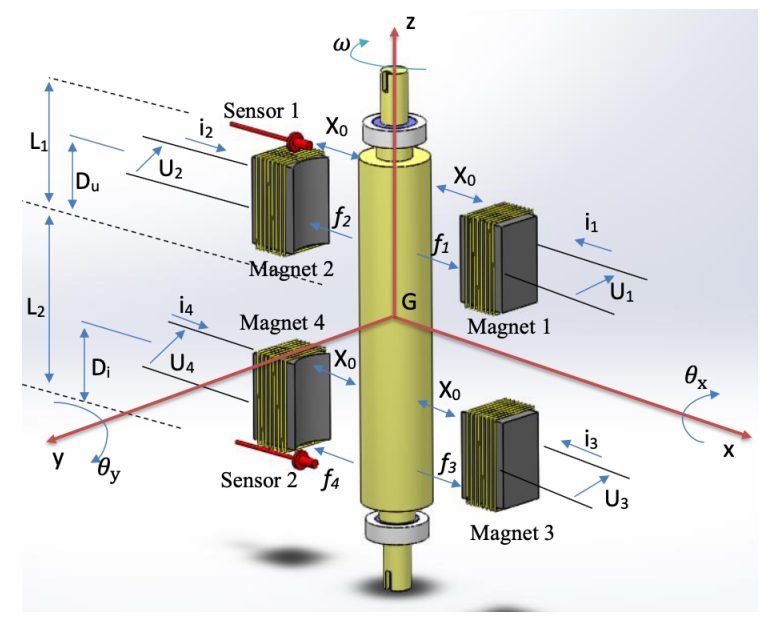

Figure 1. Four electromagnet system

Each pair, then produce the forces of attraction $F_{1} \& F_{2}$ and $F_{3} \& F_{4}$ which are adjusted by regulating the currents $i_{1}, i_{2}, i_{3}$ and $i_{4}$ respectively so that the shaft can be kept balance in the space within those magnets. Assuming that $\left(x_{1}, i_{1}\right),\left(x_{2}, i_{2}\right),\left(x_{3}, i_{3}\right),\left(x_{4}, i_{4}\right)$ are the positions and currents of electromagnets $1,2,3$, and 4 respectively. The expression of the magnetic forces is given as

$$
\begin{aligned}
& F_{1}=\frac{\mu_{g} N^{2} i_{1}^{2} A_{g}}{4 x_{1}^{2}}=\frac{K}{4}\left(\frac{i_{1}}{x_{1}}\right)^{2}, F_{2}=\frac{\mu_{g} N^{2} i_{2}^{2} A_{g}}{4 x_{2}^{2}}=\frac{K}{4}\left(\frac{i_{2}}{x_{2}}\right)^{2} \\
& F_{3}=\frac{\mu_{g} N^{2} i_{3}^{2} A_{g}}{4 x_{3}^{2}}=\frac{K}{4}\left(\frac{i_{3}}{x_{3}}\right)^{2}, F_{4}=\frac{\mu_{g} N^{2} i_{4}^{2} A_{g}}{4 x_{4}^{2}}=\frac{K}{4}\left(\frac{i_{4}}{x_{4}}\right)^{2}
\end{aligned}
$$

where $\mathrm{K}$ is a coefficient and calculated as $K=\mu_{g} N^{2} A_{g}$, where $\mu_{g}$ is the permeability of air, $N$ is the number of turns in each coil, and $A_{g}$ is the cross-section area of the electromagnet. It is assumed that the inertia and geometric rotating axes of the rigid rotor coincide to each other, hence, the central point $G$ is the mass center of the rotor and $m$ as its mass. its mass. The $x$ axis direction forces (1) exerted on rotor result in translational and rotational motions such that $x$ and $\theta_{y}$ DOF's force and torque equations are given as (2) and (3) respectively:

$$
m\left(\ddot{x}_{g}\right)=\left(F_{1}-F_{2}\right)+\left(F_{3}-F_{4}\right)
$$




$$
I_{r} \cdot \ddot{\theta}_{y}=-I_{a} \cdot \omega \cdot \dot{\theta}_{x}+\left(F_{1}-F_{2}\right) \cdot D_{u}-\left(F_{3}-F_{4}\right) \cdot D_{l}
$$

Where $I_{a}$ and $I_{r}$ are the total moments of inertia about axial and radial direction axes $z, x$ and $y$ through the rotor's mass centre or center of weigth, respectively, $\theta_{y}$ is the rotor angle over y axis, $D_{u}$ is the distance form the top electromagnets to rotor central $G, D_{l}$ is the distance form the bottom electromagnets to rotor central $G,-I_{a} \cdot \omega \cdot \dot{\theta}_{x}$ is the reinforced torque of rotor, as shown in Figure 1. If the distance between rotor and the magnet at stable position is $x_{0}$, movement of rotor within the top two magnets is $x_{u}$ and that within the bottom two magnets is $x_{l}$, the distances $x_{1}, x_{2}, x_{3}, x_{4}$ between rotor and each magnet can be calculated as with respect to the top two magnets: $\left\{\begin{array}{l}x_{1}=x_{0}-x_{u} \\ x_{2}=x_{0}+x_{u}\end{array}\right.$ and with respect to the bottom two magnets: $\left\{\begin{array}{l}x_{3}=x_{0}-x_{l} \\ x_{4}=x_{0}+x_{l}\end{array}\right.$. It is found that the AMB 4th order system with two pairs of electromagnets arranged as in Figure 1 can be separated into two magnet systems, or simplified to two 2nd order systems. In that case, with an assumption of very small $\theta_{y}$, the movement of rotor is represented as, wrt. the two upper magnets:

$$
x_{u}=x_{g}+D_{u} \theta_{y}
$$

and for the two lower magnets:

$$
x_{l}=x_{g}-D_{l} \theta_{y}
$$

Taking 2nd order derivatives of (4), and combining with (2) and (3), we have

$$
\begin{aligned}
\ddot{x}_{u} & =\ddot{x}_{g}+D_{u} \ddot{\theta}_{y} \\
& =\frac{1}{m} F_{1}-\frac{1}{m} F_{2}+D_{u}\left(\frac{1}{I_{r}} F_{1} D_{u}-\frac{1}{I_{r}} F_{2} D_{u}\right) \\
& =a_{u}\left[\frac{i_{1}^{2}}{\left(x_{0}-x_{u}\right)^{2}}-\frac{i_{2}^{2}}{\left(x_{0}+x_{u}\right)^{2}}\right]
\end{aligned}
$$

where $a_{u}=\frac{K_{u}}{4}\left(\frac{1}{m}+\frac{D_{u}^{2}}{I_{r}}\right)$. It is noted that the coupling term related to $\theta_{x}$ and $\theta_{y}$ is omitted. The coupling effects is considered as system disturbances. Applying the same procedure, from (5):

$$
\begin{aligned}
\ddot{x}_{l} & =\ddot{x}_{g}-D_{l} \ddot{\theta}_{y} \\
& =a_{l}\left[\frac{i_{3}^{2}}{\left(x_{0}-x_{l}\right)^{2}}-\frac{i_{4}^{2}}{\left(x_{0}+x_{l}\right)^{2}}\right]
\end{aligned}
$$

where $a_{l}=\frac{K_{l}}{4}\left(\frac{1}{m}+\frac{D_{l}^{2}}{I_{r}}\right)$. On the other hand, applying Kirchhoff's voltage law for each coil, we have the following equations:

$$
\begin{aligned}
& u_{1}=R i_{1}+L_{s} \frac{d i_{1}}{d t}+\frac{K}{2} \frac{d}{d t}\left(\frac{i_{1}}{x_{1}}\right), u_{2}=R i_{2}+L_{s} \frac{d i_{2}}{d t}+\frac{K}{2} \frac{d}{d t}\left(\frac{i_{2}}{x_{2}}\right) \\
& u_{3}=R i_{3}+L_{s} \frac{d i_{3}}{d t}+\frac{K}{2} \frac{d}{d t}\left(\frac{i_{3}}{x_{3}}\right), u_{4}=R i_{4}+L_{s} \frac{d i_{4}}{d t}+\frac{K}{2} \frac{d}{d t}\left(\frac{i_{4}}{x_{4}}\right)
\end{aligned}
$$

Deriving form (8) and (9), the currents are represented as:

$$
\begin{aligned}
& \dot{i}_{1}=\frac{2\left(x_{0}-x_{u}\right)}{2 L_{s}\left(x_{0}-x_{u}\right)+K}\left(u_{1}-R i_{1}-\frac{K . v_{u} i_{1}}{2\left(x_{0}-x_{u}\right)^{2}}\right), \dot{i}_{2}=\frac{2\left(x_{0}+x_{u}\right)}{2 L_{s}\left(x_{0}+x_{u}\right)+K}\left(u_{2}-R i_{2}+\frac{K . v_{u} i_{2}}{2\left(x_{0}+x_{u}\right)^{2}}\right), \\
& \dot{i}_{3}=\frac{2\left(x_{0}-x_{l}\right)}{2 L_{s}\left(x_{0}-x_{l}\right)+K}\left(u_{3}-R i_{3}-\frac{K \cdot v_{l} i_{3}}{2\left(x_{0}-x_{l}\right)^{2}}\right), \dot{i}_{4}=\frac{2\left(x_{0}+x_{l}\right)}{2 L_{s}\left(x_{0}+x_{l}\right)+K}\left(u_{4}-R i_{4}+\frac{K \cdot v_{l} i_{4}}{2\left(x_{0}+x_{l}\right)^{2}}\right),
\end{aligned}
$$


In (4), (10), together presents the mathematical model of the two upper magnets as below:

$$
\left\{\begin{array}{l}
\dot{x}_{u}=v_{u} \\
\dot{v}_{u}=a_{u}\left(\frac{i_{1}}{x_{0}-x_{u}}\right)^{2}-a_{u}\left(\frac{i_{2}}{x_{0}+x_{u}}\right)^{2} \\
\dot{i}_{1}=\frac{2\left(x_{0}-x_{u}\right)}{2 L_{s}\left(x_{0}-x_{u}\right)+K}\left(u_{1}-R i_{1}-\frac{K v_{u} i_{1}}{2\left(x_{0}-x_{u}\right)^{2}}\right) \\
\dot{i}_{2}=\frac{2 \cdot\left(x_{0}+x_{u}\right)}{2 L_{s}\left(x_{0}+x_{u}\right)+K}\left(u_{2}-R i_{2}+\frac{K v_{u} i_{2}}{2 \cdot\left(x_{0}+x_{u}\right)^{2}}\right)
\end{array}\right.
$$

Similarly, (5), (11) provides the mathematical model of the two lower magnets:

$$
\left\{\begin{array}{l}
\dot{x}_{l}=v_{l} \\
\dot{v}_{l}=a_{l}\left(\frac{i_{3}}{x_{0}-x_{l}}\right)^{2}-a_{l}\left(\frac{i_{4}}{x_{0}+x_{l}}\right)^{2} \\
\dot{i}_{3}=\frac{2\left(x_{0}-x_{l}\right)}{2 L_{s}\left(x_{0}-x_{l}\right)+K}\left(u_{3}-R i_{3}-\frac{K v_{l} i_{3}}{2\left(x_{0}-x_{l}\right)^{2}}\right) \\
\dot{i}_{4}=\frac{2\left(x_{0}+x_{l}\right)}{2 L_{s}\left(x_{0}+x_{l}\right)+K}\left(u_{4}-R i_{4}+\frac{K v_{l} i_{4}}{2\left(x_{0}+x_{l}\right)^{2}}\right)
\end{array}\right.
$$

In summary, the system model of AMB studied in this work consists of (12) and (13) and will be used in the subsequent sections to design controller and investigate the operation.

\section{CONTROLLER DESIGN}

It is assumed that the speed can be estimated as derivative of position, the effect of rotor speed on system operation is negligible, and magnetizing currents are taken as control input.

\subsection{Control law of the two upper electromagnets}

Step 1: Find the controller to enable the position $x_{u}$ of the rotor track desired set point at the stable value which is 0 (along $0 \mathrm{x}$ axis). If $z_{1}$ is the difference between the rotor position and the stable one: $z_{1}=x_{u}$, and its derivative is $\dot{z}_{1}=\dot{x}_{u}=v_{u}$. Considering the following barrier Lyapunov candidate function:

$$
V_{1}=\frac{1}{2} \ln \frac{k_{b}^{2}}{k_{b}^{2}-z_{1}^{2}}
$$

where $k_{b}$ is the limit of $z_{1}$. It is clear that $V_{1}\left(z_{1}\right)$ is radially unbounded as $z_{1}$ approaches $k_{b}$ or $-k_{b}$. The Barrier Lyapunov function is used to reduce the error in rotor shaft position when comparing with the desired value, so as it would prevent the rotor shaft move too far away with a large distance which is greater than the air gap. This would lead to the collision between the rotor and the magnets, then damage the system. The derivative of (14) is

$$
\dot{V}_{1}=\frac{z_{1} \cdot \dot{z}_{1}}{k_{b}^{2}-z_{1}^{2}}
$$

Based on Lyapunov stability, it is required that $\dot{V}_{1} \leq 0$, thus virtual control function can be selected as

$$
v_{u d k}=-\left(k_{b}^{2}-z_{1}^{2}\right) k_{1} z_{1}
$$

where $k_{1}$ is a positive constant. Then, $\dot{V}_{1}=\frac{z_{1} v_{u d k}}{k_{b}^{2}-z_{1}^{2}}=-k_{1} z_{1}^{2} \leq 0$ satisfies the stable condition. Let $v_{u d k}=\alpha_{1}$, we have:

$$
\dot{v}_{u d k}=\dot{\alpha}_{1}=\frac{\partial \alpha_{1}}{\partial z_{1}} \dot{z}_{1}=\left(-k_{1} k_{b}^{2}+3 k_{1} z_{1}^{2}\right) \dot{z}_{1}
$$


Thus, $v_{u}$ is the virtual control which facilitates $x_{u}$ reach the set points.

Step 2: Identify virtual control to regulate $v_{u}$ to match $v_{u d k}$. If deviation of $v_{u}$ from $v_{u d k}$ is $z_{2}$ :

$$
z_{2}=v_{u}-v_{u d k}=v_{u}-\alpha_{1}
$$

Or it can be represented as $v_{u}=v_{u d k}+z_{2}$. Derivative of (18) results in:

$$
\dot{z}_{2}=\dot{v}_{u}-\dot{\alpha}_{1}=\dot{v}_{u}-\frac{\partial \alpha_{1}}{\partial z_{1}} \dot{x}_{u}
$$
side to get:

The Lyapunov candidae function in this step is chosen as: $V_{2}=V_{1}+\frac{1}{2} z_{2}^{2}$, we then differentiate both

$$
\dot{V}_{2}=-k_{1} z_{1}^{2}+\frac{z_{1} z_{2}}{k_{b}^{2}-z_{1}^{2}}+z_{2}\left(\dot{v}_{u}-\dot{\alpha}_{1}\right)
$$

In order to have $\dot{V}_{2} \leq 0$, the virtual control function is selected as $\dot{v}_{u d k}=\alpha_{2}=-k_{2} z_{2}+\dot{\alpha}_{1}-\frac{z_{1}}{k_{b}^{2}-z_{1}^{2}}$, where $k_{2}$ is a positive constant. Substitute $\dot{v}_{u d k}$ in (3.1.) for $\dot{v}_{u}$ in (20), we have:

$$
\begin{aligned}
\dot{V}_{2} & =-k_{1} z_{1}^{2}+\frac{z_{1} z_{2}}{k_{b}^{2}-z_{1}^{2}}+z_{2}\left(-k_{2} z_{2}+\dot{\alpha}_{1}-\frac{z_{1}}{k_{b}^{2}-z_{1}^{2}}-\dot{\alpha}_{1}\right) \\
& =-k_{1} z_{1}^{2}-k_{2} z_{2}^{2}
\end{aligned}
$$

In (21) shows that $\dot{V}_{2} \leq 0$ as required for stability. Therefore, $\dot{v}_{u}$ as virtual control law is identified. It is a function of $i_{1}$ and $i_{2}$ based on (12):

$$
\dot{v}_{u}=a_{u} \cdot\left(\frac{i_{1}}{x_{0}-x_{u}}\right)^{2}-a_{u} \cdot\left(\frac{i_{2}}{x_{0}+x_{u}}\right)^{2}
$$

Let $\alpha_{u}=\alpha_{2} / a_{u}$, from the above equation, it can be shown that $\dot{\alpha}_{u}=\frac{\partial \alpha_{u}}{\partial z_{1}} \dot{z}_{1}+\frac{\partial \alpha_{u}}{\partial v_{u}} \dot{v}_{u}$

Step 3: Design the current control law such that current $i$ would match the set point $i_{d}$. As presented in Step 2, the virtual control law $\dot{v}_{u}$ is a function of 2 currents $i_{1}$ and $i_{2}$, which are equivalent to electromagnetic forces of the two magnets. The fact that these two magnets operate simultaneously to maintain electromagnetic forces leads to higher energy consumption. Thus, a control scheme of switching on and off the two currents sequentially is employed to achieve energy savings as the following:

Case 1: $x_{u}<0$ and $i_{2}=0$, it is shown that

$$
i_{1 d}=\left(x_{0}-x_{u}\right) \sqrt{\alpha_{u}}
$$

On the other hand: $\dot{i}_{1 d}=\frac{\partial i_{1 d}}{\partial z_{1}} \dot{z}_{1}+\frac{\partial i_{1 d}}{\partial v_{u}} \dot{v}_{u}$, where $\dot{v}_{u}=a_{u} \cdot\left(\frac{i_{1}}{x_{0}-x_{u}}\right)^{2}$. Call $z_{v 1}$ is the deviation between $i_{1}$ and set point $i_{1 d}$, i.e.: $z_{v 1}=i_{1}-\dot{i}_{1 d}$. Differentiating both side of Equation 3.1., we get: $\dot{z}_{v 1}=$ $\dot{i}_{1}-\dot{i}_{1 d}$. In order to limit the input signal, i.e. current, within a bounded range, the current variable is provided as

$$
\left\{\begin{array}{l}
\dot{i}_{1}=I_{1} \\
i_{1}=i_{m} \tanh \left(\frac{v}{i_{m}}\right)
\end{array}\right.
$$

where $i_{m}$ is the magnitude of current range, and $v$ is the coefficient of $\tanh ()$. Consider the Lyapunov candidate function in this step as: $V_{3}=V_{2}+\frac{1}{2} z_{v 1}^{2}$, the derivative of this Equation is:

$$
\dot{V}_{3}=\dot{V}_{2}+z_{v 1}\left(I_{1}-\frac{\partial i_{1 d}}{\partial z_{1}} \dot{z}_{1}-\frac{\partial i_{1 d}}{\partial v_{u}} \dot{v}_{u}\right)
$$

Based on (25) and the condition that $\dot{V}_{3} \leq 0$, the control law $I_{1}$ is selected as

$$
I_{1}=-k_{v 1} z_{v 1}+\frac{\partial i_{1 d}}{\partial z_{1}} \dot{z}_{1}+\frac{\partial i_{1 d}}{\partial v_{u}} \dot{v}_{u}
$$


where $k_{v 1}$ is a positive constant. Substitute this $I_{1}$ in (25), we have

$$
\begin{aligned}
\dot{V}_{3} & =\dot{V}_{2}+z_{v 1}\left(-k_{v 1} z_{v 1}+\frac{\partial i_{1 d}}{\partial z_{1}} \dot{z}_{1}+\frac{\partial i_{1 d}}{\partial v_{u}} \dot{v}_{u}\right)-\frac{\partial i_{1 d}}{\partial z_{1}} \dot{z}_{1}-\frac{\partial i_{1 d}}{\partial v_{u}} \dot{v}_{u} \\
& =\dot{V}_{2}-k_{v 1} z_{v 1}^{2}
\end{aligned}
$$

It can be seen from (27) that $\dot{V}_{3} \leq 0$ which satisfies stable condition. Thus, with $x_{u}>0, I_{1}$ as in (26) is the control law to stabilize the upper part of the rotor.

Case 2: $x_{u}>0$ with respect to $i_{1}=0$. The condition implies that: $i_{2 d}=\left(x_{0}+x_{u}\right) \sqrt{-\alpha_{u}}$ where $\dot{v}_{u}=-a_{u} \cdot\left(\frac{i_{2}}{x_{0}+x_{u}}\right)^{2}$. Call $z_{v 2}$ is the deviation between $i_{2}$ and set point $i_{2 d}: z_{v 2}=i_{2}-\dot{i}_{2 d}$. Differentiating both side of (3.1.), we get $\dot{z}_{v 2}=\dot{i}_{2}-\dot{i}_{2 d}$. Similar to case 1 :

$$
\left\{\begin{array}{l}
\dot{i}_{2}=I_{2} \\
i_{2}=i_{m} \tanh \left(\frac{v}{i_{m}}\right)
\end{array}\right.
$$

The Lyapunov candidate function in this case is $V_{4}=V_{2}+\frac{1}{2} z_{v 2}^{2}$. The derivative of this Lyapunov function is:

$$
\dot{V}_{4}=\dot{V}_{2}+z_{v 2}\left(I_{2}-\frac{\partial i_{2 d}}{\partial z_{1}} \dot{z}_{1}-\frac{\partial i_{2 d}}{\partial v_{u}} \dot{v}_{u}\right)
$$

With the condition of $\dot{V}_{4} \leq 0$, the control function $I_{2}$ is selected as:

$$
I_{2}=-k_{v 2} z_{v 2}+\frac{\partial i_{2 d}}{\partial z_{1}} \dot{z}_{1}+\frac{\partial i_{2 d}}{\partial v_{u}} \dot{v}_{u}
$$

where $k_{v 2}$ is a positive constant. Substitute the selected $I_{2}$ in (29), we have:

$$
\begin{aligned}
\dot{V}_{4} & =\dot{V}_{2}+z_{v 2}\left(-k_{v 2} z_{v 2}+\frac{\partial i_{2 d}}{\partial z_{1}} \dot{z}_{1}+\frac{\partial i_{2 d}}{\partial v_{u}} \dot{v}_{u}\right)-\frac{\partial i_{2 d}}{\partial z_{1}} \dot{z}_{1}-\frac{\partial i_{2 d}}{\partial v_{u}} \dot{v}_{u} \\
& =\dot{V}_{2}-k_{v 2} z_{v 2}^{2}
\end{aligned}
$$

In (31) shows obviously that $\dot{V}_{4} \leq 0$ which satisfies stable condition, and the control law $I_{2}$ selected can stabilize the upper part of the rotor.

\subsection{Control law of the two lower electromagnets}

The design procedure is similar to that of two upper electromagnets as presented in a). It also includes 3 steps as the following:

Step 1: Identify position control $x_{l}$ to reach the stable position, which is 0 (along $0 x$ axis). Let $z_{3}$ be the deviation between rotor shaft and the stable position, i.e.: $z_{3}=x_{1} \Rightarrow \dot{z_{3}}=\dot{x}_{l}=v_{l}$ The barrier Lyapunov candidate function is $V_{5}=\frac{1}{2} \ln \frac{k_{b}^{2}}{k_{b}^{2}-z_{3}^{2}}$. The virtual control is chosen as $v_{l d k}=-\left(k_{b}^{2}-z_{3}^{2}\right) k_{3} z_{3}$ where $k_{3}$ is a positive constant. Similarly, it can be proven that this control law renders $\dot{V}_{5} \leq 0$. Let $v_{l d k}=\alpha_{3}$, and compute its time derivative

$$
\dot{v}_{l d k}=\dot{\alpha}_{3}=\frac{\partial \alpha_{3}}{\partial z_{3}} \dot{z}_{3}=\left(-k_{3} k_{b}^{2}+3 k_{3} z_{3}^{2}\right) \dot{z}_{3}
$$

Step 2: Select virtual control so that $v_{l}$ would be able to reach $v_{l d k}$. Let the difference between $v_{l}$ and $v_{l d k}$ be $z_{4}: z_{4}=v_{l}-v_{l d k}=v_{l}-\alpha_{3}$. Or $v_{l}=v_{l d k}+z_{3}$ In this step, the Lyapunov candidate function is $V_{6}=V_{5}+\frac{1}{2} z_{4}^{2}$. We pick the virtual control to satisfy that $\dot{V}_{6} \leq 0$ as:

$$
\dot{v}_{l d k}=\alpha_{4}=-k_{4} z_{4}+\dot{\alpha}_{3}-\frac{z_{3}}{k_{b}^{2}-z_{3}^{2}}
$$


where $k$ is a positive constant. In (13) provides the calculation of $\dot{v}_{l}$ from $i_{3}$ and $i_{4}$ as:

$$
\dot{v}_{l}=a_{l} \cdot\left(\frac{i_{3}}{x_{0}-x_{l}}\right)^{2}-a_{l} \cdot\left(\frac{i_{4}}{x_{0}+x_{l}}\right)^{2}
$$

Let $\alpha_{l}=\alpha_{3} / a_{l}$, we have $\dot{\alpha}_{l}=\frac{\partial \alpha_{l}}{\partial z_{3}} \dot{z}_{3}+\frac{\partial \alpha_{l}}{\partial v_{l}} \dot{v}_{l}$

Step 3: The switching scheme of currents supplied to lower magnets are

Case 1: $x_{l}<0$ and $i_{4}=0$ implies that: $i_{3 d}=\left(x_{0}-x_{l}\right) \sqrt{\alpha_{l}}$ And thus, $\dot{i}_{3 d}=\frac{\partial i_{3 d}}{\partial z_{3}} \dot{z}_{3}+\frac{\partial i_{3 d}}{\partial v_{l}} \dot{v}_{l}$. Let $z_{v 3}$ is the deviation of $i_{3}$ from set point $i_{3 d}$, we have: $z_{v 3}=i_{3}-\dot{i}_{3 d}$. Using tanh to limit $i_{3}$ in the required range:

$$
\left\{\begin{array}{l}
\dot{i}_{3}=I_{3} \\
i_{3}=i_{m} \tanh \left(\frac{v}{i_{m}}\right)
\end{array}\right.
$$

Barrier Lyapunov function in this step is $V_{7}=V_{6}+\frac{1}{2} z_{v 3}^{2}$. In order to render $\dot{V}_{7} \leq 0$, virtual control $I_{3}$ is selected as

$$
I_{3}=-k_{v 3} z_{v 3}+\frac{\partial i_{3 d}}{\partial z_{3}} \dot{z}_{3}+\frac{\partial i_{3 d}}{\partial v_{l}} \dot{v}_{l}
$$

where $k_{v 3}$ is a positive constant.

Case 2: $x_{l}>0$ and $i_{3}=0$ yields $i_{4 d}=\left(x_{0}+x_{l}\right) \sqrt{-\alpha_{l}}$ and its derivatives is $\dot{i}_{4 d}=\frac{\partial i_{4 d}}{\partial z_{3}} \dot{z}_{3}+\frac{\partial i_{4 d}}{\partial v_{l}} \dot{v}_{l}$, where $\dot{v}_{l}=-a_{l} \cdot\left(\frac{i_{4}}{x_{0}+x_{l}}\right)^{2}$. Applying the analogous design. then selecting virtual control $I_{4}$ is shown as below

$$
I_{4}=-k_{v 4} z_{v 4}+\frac{\partial i_{4 d}}{\partial z_{3}} \dot{z}_{3}+\frac{\partial i_{4 d}}{\partial v_{l}} \dot{v}_{l}
$$

where $k_{v 4}$ is a positive constant.

\section{SIMULATION AND DISCUSSION}

Numerical simulation parameters used in the study are presented as: Rotor mass $m=5 \mathrm{~kg}$; number of coil turns $N=400$ turns; nominal air gap $x_{0}=0.001 \mathrm{~m}$; maximum position error $k_{b}=0.001 \mathrm{~m}$; initial position of upper rotor shaft $x_{u}=0.0001 \mathrm{~m}$; initial position of lower rotor shaft $x_{l}=0.0001 \mathrm{~m}$; self inductance $L_{s}$ is $0.001 \mathrm{H}$; cross section area of iron core $A_{g}$ is $0.001 \mathrm{~m}^{2}$; permeability of air gap $\mu_{g}=1.256 \times 10^{-6} \mathrm{H} / \mathrm{m}$; moment of inertia $I_{r}=2.900 \times 10^{-2} \mathrm{~kg} \cdot \mathrm{m}^{2}$; distance from rotor central to upper magnets $D_{u}=4.166 \times 10^{-2} \mathrm{~m}$; distance from rotor central to lower magnets $D_{l}=7.602 \times 10^{-2} \mathrm{~m}$. Controller's coefficients are of $k_{1}=11 ; k_{2}=1700 ; k_{v 1}=$ $700 ; k_{v 2}=10000 ; k_{3}=10 ; k_{4}=1600 ; k_{v 3}=700 ; k_{v 4}=10000$. In the paper, to emphasize the ability of handling input and output constraints of the proposed controller, the rotor shaft is driven to equilibrium position and accelerating to $1000 \mathrm{rpm}$. This simulation procedure implies the effects of coupling term related to $\theta_{x}$ and $\theta_{y}$ can be eliminated.

Case study 1: current limit is $i_{m}=3 \mathrm{~A}$. As shown in Figure 2a and 2b, the upper and lower body of the AMB can be regulated from its deviation to the stable position within 0.01 second. Duration to reach the zero displacement lower body is also around 0.01 second, however the overshoot is a little bit more, i.e. around $5 \mu \mathrm{m}$, it is clear that the value is well below the threshold define by $k_{b}$. Meanwhile the duration of central displacement is corrected within the same interval and the overshoot is slightly smaller than that of lower body.

Case study 2: current limit is $i_{m}=2 \mathrm{~A}$. The current limit is reduced to $2 \mathrm{~A}$ in this case, but the initial displacement of the rotor shaft is kept the same. It is clearly observed that the settling times of the upper body and lower body in Figure $2 \mathrm{a}$ and Figure $2 \mathrm{~b}$ are slightly longer that those in case one in Figure $3 \mathrm{a}, 3 \mathrm{~b}$ respectively. It is due to the fact that controllers need to take more effort to stabilise the system with smaller current fed thanks to the use of the hyperbolic tangent function in the design. The peak currents supplied to the AMB electromagnetics are all less than the provided limit as shown in both two cases as illustrated in Figure 4 and Figure 5. These peak values for lower magnets are also less than those of the upper ones. When the current limit is decreased, it is observed that there is more oscillation of current response. The cause can be explained as less magnetic forces provided to the system. 


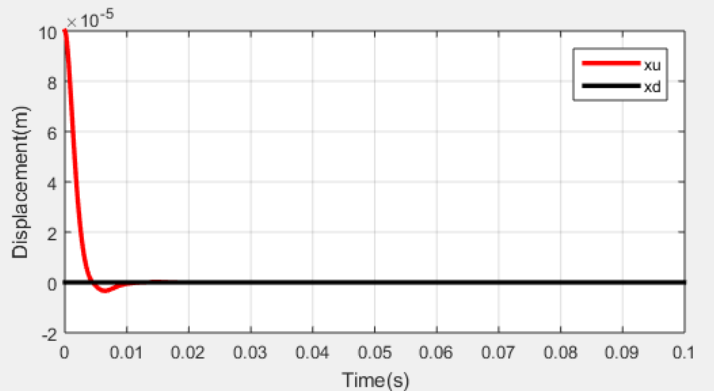

(a)

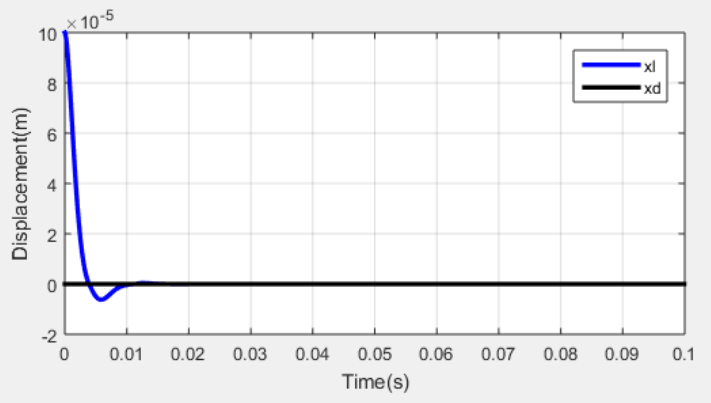

(b)

Figure 2. Rotor displacement, (a) Upper body displacement, and (b) Lower body displacement

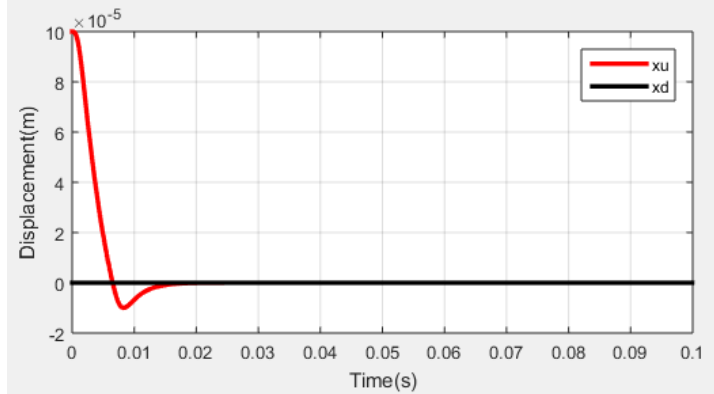

(a)

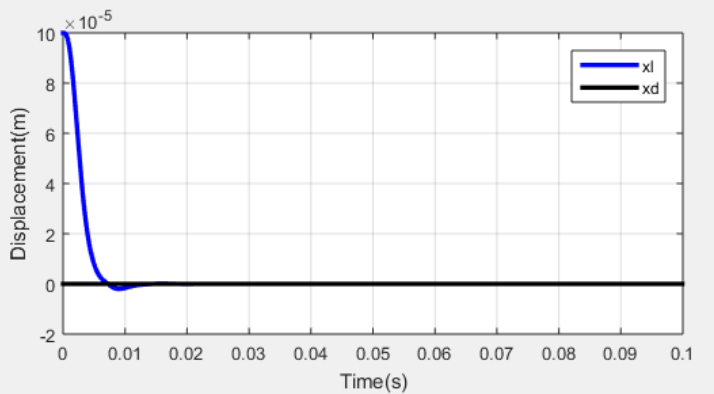

(b)

Figure 3. Rotor displacement, (a) Upper body displacement, and (b) Lower body displacement

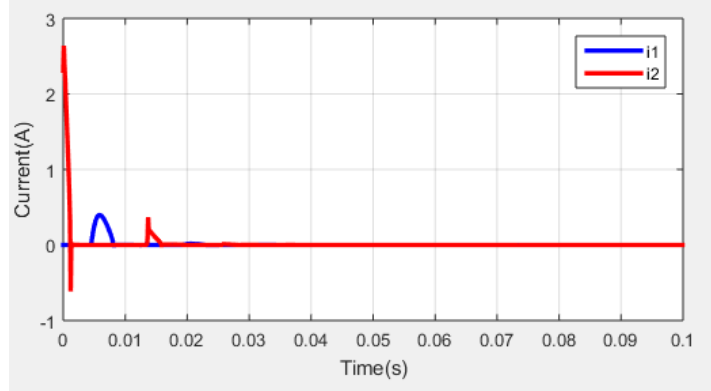

(a)

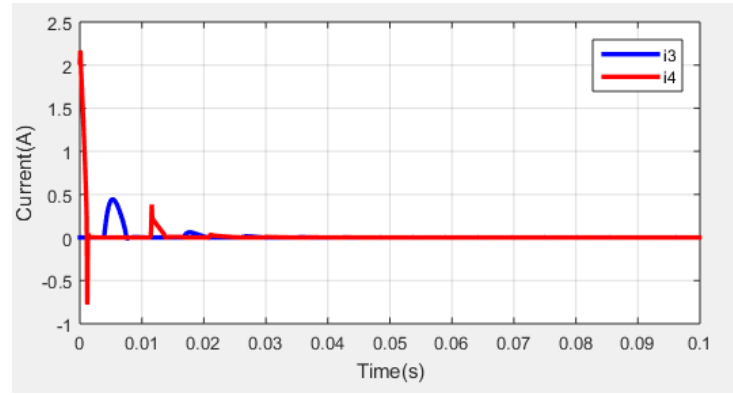

(b)

Figure 4. Current responses, (a) Upper magnets, and (b) Lower magnets

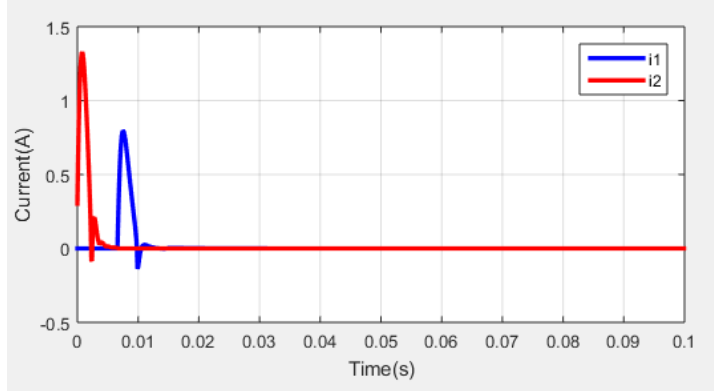

(a)

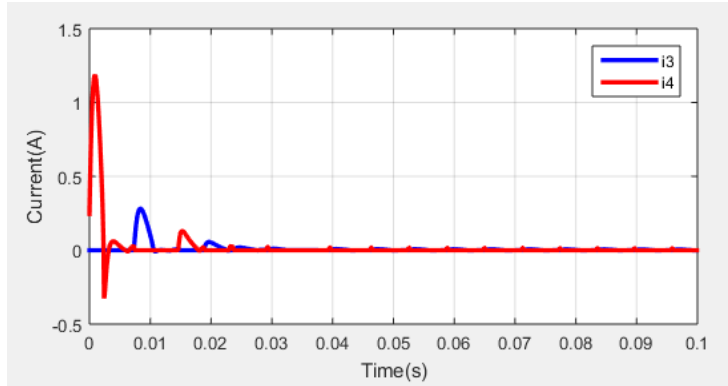

(b)

Figure 5. Current responses, (a) Upper magnets, and (b) Lower magnets 


\section{CONCLUSIONS}

In this paper, a 4th order AMB has been modeled as two 2nd order subsystem with magnetizing current is treated as control input. The backstepping method is adopted in control design for the obtained model. The controllers have been built and validated via simulation in different case studies in a view of input saturation and bounded output. It is shown that our proposed approach is able to facilitate the AMB regulate gap deviations as desire and thus stabilizes the system. Future work include practical implementation of the whole system, it would enable further investigation of the proposed works thoroughly for real-life applications.

\section{ACKNOWLEDGEMENTS}

This research was funded by Hanoi University of Science and Technology grant number T2018-PC057.

\section{REFERENCES}

[1] N. F. Al-Muthairi and M. Zribi, "Sliding mode control of a magnetic levitation system," Mathematical Problems in Engineering, vol. 2004, no. 2, pp. 93-107, 2004.

[2] E. Maslen and G. Schweitzer, Magnetic Bearings-Theory, Design and Application to Rotating Machinery, Berlin: Springer, vol. 1, 2009.

[3] F. R. Ismagilov and V. E. Vavilov, "Superhigh-speed electric motor with unipolar magnetic bearing," Russian Engineering Research, vol. 38, no. 6, pp. 480-484, 2018.

[4] E. Gourc, S. Seguy, and L. Arnaud, "Chatter milling modeling of active magnetic bearing spindle in highspeed domain,” International Journal of Machine Tools and Manufacture, vol. 51, no. 12, pp. 928-936, 2011.

[5] C. R. Knospe, "Active magnetic bearings for machining applications," Control Engineering Practice, vol. 15, no. 3, pp. 307-313, 2007.

[6] B.Han, Z.Huang, and Y.Le, "Design aspects of a large scale turbomolecular pump with active magnetic bearings," Vacuum, vol. 142, pp. 96-105, 2017.

[7] T. Allison, J. Moore, R. Pelton, J. Wilkes, and B. Ertas, “7 - turbomachinery," Fundamentals and Applications of Supercritical Carbon Dioxide (sCO2) Based Power Cycles, K. Brun, P. Friedman, and R. Dennis, Eds. Woodhead Publishing, pp. 147-215, 2017.

[8] D. Clark, M. Jansen, and G. Montague, "An overview of magnetic bearing technology for gas turbine engines," NASA Technical Reports Server (NTRS), 2004.

[9] W. Zhang and H. Zhu, "Radial magnetic bearings: An overview," Results in Physics, vol. 7, pp. 37563766, 2017.

[10] L.-C. Lin and T.-B. Gau, "Feedback linearization and fuzzy control for conical magnetic bearings," IEEE transactions on control systems technologyvol., vol. 5, no. 4, pp. 417-426, 1997.

[11] N. Sarmah and R. Tiwari, "Identification of crack and internal damping parameters using full spectrum responses from a jeffcott rotor incorporated with an active magnetic bearing," International Conference on Rotor Dynamics., pp. 34-48, 2019.

[12] Q. Li, W. Wang, B. Weaver, and X. Shao, "Active rotordynamic stability control by use of a combined active magnetic bearing and hole pattern seal component for back-to-back centrifugal compressors," Mechanism and Machine Theory, vol. 127, pp. 1-12, 2018.

[13] J. Sun, H. Zhou, X. Ma, and Z. Ju, "Study on pid tuning strategy based on dynamic stiffness for radial active magnetic bearing," ISA Transactions, vol. 80, pp. 458-474, 2018.

[14] A. M. A.-H. Shata, R. A. Hamdy, A. S. Abdelkhalik, and I. El-Arabawy, "A fractional order pid control strategy in active magnetic bearing systems," Alexandria Engineering Journal, vol. 57, no. 4, pp. 39853993, 2018.

[15] M. S. Kang, J. Lyou, and J. K. Lee, "Sliding mode control for an active magnetic bearing system subject to base motion," Mechatronics, vol. 20, no. 1, pp. 171-178, 2010.

[16] A. Bonfitto, L. M. Castellanos Molina, A. Tonoli, and N. Amati, "Offset-Free Model Predictive Control for Active Magnetic Bearing Systems," Actuators, vol. 7, no. 3, p. 46, 2018.

[17] R. HE and K.-Z. LIU, "A Nonlinear Output Feedback Control Method for a 5DOF Active Magnetic Bearing System," Transactions of the Society of Instrument and Control Engineers, vol. 41, no. 3, pp. 216-225, 2014. 
[18] Y. LI, Q. ZHANG, and X.-W. WANG, "The Terminal Sliding Mode Control of 4-DoF Active Magnetic Bearing Rotor System," DEStech Transactions on Engineering and Technology Research, no. icmm, pp. 139-143, 2018.

[19] S.-L. CHEN and Y.-H. HSIAO, "Smooth voltage controller and observer for a three-pole active magnetic bearing system,” Mechanical Engineering Journal, vol. 4, no. 5, pp. 16-00 718-16-00 718, 2017.

[20] N. P. Q. Vo Thanh Ha, Le Trong Tan, Nguyen Duc Nam, "Backstepping Control of Two-Mass System Using Induction Motor Drive Fed by Voltage Source Inverter with Ideal Control Performance of Stator Current," International Journal of Power Electronics and Drive System, vol. 10, no. 2, pp. 720-730, 2019.

[21] I. A. Raptis and K. P. Valavanis, "Linear and Nonlinear Control of Small-Scale Unmanned Helicopters," Springer Science Business Media, 2010.

[22] C. Hua, P. X. Liu, and X. Guan, "Backstepping control for nonlinear systems with time delays and applications to chemical reactor systems," IEEE Transactions on Industrial Electronics., vol. 56, pp. 3723-3732, 2009.

[23] M. LIU, S. Xu, and C. Han, "A backstepping simple adaptive control application to flexible space structures," Chinese Journal of Aeronautics, vol. 25, p. 446452, 062012.

[24] A. Golubev, A. Krishchenko, and N. Utkina, "Missile angle of attack tracking using integrator backstepping," IFAC-PapersOnLine, vol. 52, pp. 724-729, 012019.

[25] D. H. Nguyen, T. L. Nguyen, M. L. Nguyen, and H. P. Nguyen, "Nonlinear Control of an Active Magnetic Bearing with Output Constraint," International Journal of Electrical and Computer Engineering (IJECE), vol. 8, no. 5, pp. 3666-3677, 2019. 